\title{
Optimal Stationary Self-Triggered Sampling for Estimation
}

\author{
Touraj Soleymani, Student Member, IEEE, Sandra Hirche, Senior Member, IEEE, \\ and John S. Baras, Life Fellow, IEEE
}

\begin{abstract}
In this paper, we study optimal stationary sampling for transmission of measurements of a stochastic process from a source encoder to a source decoder through a costly communication channel. We measure information transferred over a time interval by the change in the decoder's entropy regarding the state of the process given the transmitted measurements. In our setting, the encoder employs a sampler to control the information flow in the channel. The problem is casted as a discounted infinite horizon optimization problem that takes into account the transferred information and the paid price. We derive the optimal stationary sampling policy, and propose two computational methods with convergence guarantees by using techniques from approximate dynamic programing. In addition, we introduce two triggering mechanisms based on the value of information and on the covariance threshold that can generate the optimal policy. Finally, we present some numerical and simulation results.
\end{abstract}

Index Terms-Approximate Policy Iteration, Approximate Value Iteration, Covariance Threshold, Self-Triggered Sampling, Optimal Stationary Policy, Value of Information.

\section{INTRODUCTION}

In this paper, we study optimal stationary sampling for transmission of measurements of a stochastic process from a source encoder to a source decoder through a costly communication channel. We measure information transferred over a time interval by the change in the decoder's entropy regarding the state of the process given the transmitted measurements. In our setting, the encoder employs a sampler to control the information flow in the channel. This study has a broad range of applications including surveillance and reconnaissance, planetary explorations, wireless wearables, teleoperation, and many other examples of cyber-physical systems.

Nonuniform sampling [1] and its important sub-class event-driven (event-triggered) sampling [2] for estimation have received early attention in the literature. Kushner [3] studies the optimal measurement control problem for linear systems subject to a constraint on the number of measurements. Meier et al. [4] look at the optimal measurement control problem subject to measurement cost, and propose dynamic programming (DP) and the gradient method as computational procedures. Åström and Bernhardsson [2] show that event-driven sampling can outperform periodic sampling

T. Soleymani and S. Hirche are with the Institute for Information-Oriented Control, Technische Universität München, D-80333 Munich, Germany (email: \{touraj, hirche\}atum.de).

J. S. Baras is with the Institute for Systems Research, University of Maryland College Park, MD 20742, USA (email: baras (umd. edu).

T. Soleymani, S. Hirche, and J. S. Baras are also with the Institute for Advanced Study, Technische Universität München, D-85748 Garching, Germany. with respect to the estimation error of a scalar linear system under a sampling rate constraint. Rabi et al. [5] study optimal event-driven sampling as a stopping time problem for a scalar system under a finite transmission budget constraint. Molin and Hirche [6] investigate the optimal design for event-driven sampling in a scalar system with communication cost by considering a two-player problem. Furthermore, Soleymani et al. [7] study optimal closed-loop sampling in which the effect of the cost-to-go is taken into account.

Event-driven sampling can reduce communication cost while providing a good estimation performance. However, it requires the encoder to monitor the process constantly. Selfdriven (self-triggered) sampling [8] is a technique in which the encoder is self-contained in decision making and can compute the sampling schedule without monitoring the process constantly. An example of a self-triggering mechanism for sampling in the estimation problem is the one based on the covariance of the estimation error. In [9], we propose an optimized self-triggering mechanism in which the parameter of the mechanism is found by solving an optimization problem. In [10], we characterize the optimal self-triggered sampling policy for the finite horizon estimation problem. In contrast, in this paper we study the infinite horizon problem. We derive the optimal stationary policy, and propose two computational methods with convergence guarantees by using techniques from approximate dynamic programing. In addition, we introduce two triggering mechanisms based on the value of information and on the covariance threshold that can generate the optimal policy.

The outline of this paper is as follows. Problem formulation is presented in Section II. In Section III, we derive the optimal stationary sampling policy, propose two computational methods for calculating this policy, and introduce two triggering mechanisms. We illustrate numerical and simulation results in Section IV. Finally, concluding remarks are made in Section V.

\section{A. Notations}

In this paper, we represent an $n$ dimensional vector with $x=\left[x_{1}, \ldots, x_{n}\right]^{T}$ where $x_{i}$ is its $i$ th component. We write $x^{T}$ to denote the transpose of the vector $x$. The identity matrix with dimension $n$ is denoted by $I_{n}$. We write $\delta_{k k^{\prime}}$ to denote the Kronecker delta function. We write $p(x)$ to denote the probability distribution of the stochastic variable $x$. The normal distribution with mean $\mu$ and covariance $\Sigma$ is denoted by $N(\mu, \Sigma)$. For matrices $A$ and $B$, we write $A \succ 0$ and $B \succeq 0$ to mean that $A$ and $B$ are positive definite and positive semi-definite, respectively. 


\section{Problem Formulation}

\section{A. Dynamical System and Filtration}

Consider a discrete-time dynamical system generated by the following linear state equation:

$$
\begin{aligned}
& x_{k}=F x_{k-1}+w_{k-1}, \\
& y_{k}=H x_{k}+v_{k},
\end{aligned}
$$

for $k=1,2, \ldots$ where $x_{k} \in \mathbb{R}^{n}$ is the state of the system at time $k, F$ is the state matrix, $w_{k} \in \mathbb{R}^{n}$ is a white noise sequence with zero mean and covariance $Q \delta_{k k^{\prime}}$ where $Q \succ 0, y_{k} \in \mathbb{R}^{p}$ is the output of the system at time $k, H$ is the output matrix, and $v_{k} \in \mathbb{R}^{p}$ is a white noise sequence with zero mean and covariance $R \delta_{k k^{\prime}}$ where $R \succ 0$. It is assumed that the initial state $x_{0}$ is a Gaussian vector with zero mean and covariance $P_{0}$, and that $x_{0}, w_{k}$, and $v_{k}$ are mutually independent. We also assume that $(F, H)$ is observable.

A source encoder samples the measurements of the process at times $k_{s}$ for $s=1, \ldots, M$ where $M$ is unknown. Samples are transmitted through a communication channel, and received by a source decoder. Through this study, the decoder assumes that measurements are never compromised.

Definition 1 (Information control): The information control $\delta_{k}$ at time $k$ is

$$
\delta_{k}= \begin{cases}1, & \text { if } \exists s: k=k_{s}, \\ 0, & \text { otherwise, }\end{cases}
$$

where $\delta_{0}=0$.

A set of information controls $\pi=\left\{\delta_{1}, \ldots, \delta_{N}\right\}$ is called a sampling policy. At each time $k$, the encoder should decide about the information control $\delta_{k}$. We assume that measurements $y_{k}$ are not explicitly used by the encoder for the decision making.

Definition 2 (Decoder's information set): The decoder's information set is the $\sigma$-algebra generated by measurements transmitted to the decoder, i.e.,

$$
\mathcal{I}_{k}=\sigma\left\{y_{l} \mid l \leq k, \delta_{l}=1\right\} .
$$

The decoder's information set is a function of the information control, i.e., $\mathcal{I}_{k}=\mathcal{I}_{k}\left(\delta_{k}\right)$. In particular, we can write

$$
\mathcal{I}_{k}\left(\delta_{k}\right)= \begin{cases}\sigma\left\{\mathcal{I}_{k-1}, y_{k}\right\}, & \text { if } \delta_{k}=1 \\ \mathcal{I}_{k-1}, & \text { otherwise }\end{cases}
$$

Filtration at the decoder is based on the decoder's information set $\mathcal{I}_{k}\left(\delta_{k}\right)$. The conditional distribution $p\left(x_{k} \mid \mathcal{I}_{k}\left(\delta_{k}\right)\right)=$ $N\left(\hat{x}_{k}, P_{k}\right)$ is a Gaussian distribution given the system's model introduced before. The conditional distribution evolves in time due to the system dynamics, and is updated at times $k_{s}$ due to measurements.

Consider the transformation $I_{k}=P_{k}^{-1}$ where $I_{k}$ is the Fisher information matrix (FIM) [11]. Following the Kolmogorov forward equation [12], the estimate and the FIM in the interval $\left(k_{s-1}, k_{s}^{-}\right]$are propagated as

$$
\begin{aligned}
\hat{x}_{k} & =F \hat{x}_{k-1}, \quad k \in\left(k_{s-1}, k_{s}^{-}\right], \\
I_{k} & =\left(F I_{k-1}^{-1} F^{T}+Q\right)^{-1}, \quad k \in\left(k_{s-1}, k_{s}^{-}\right],
\end{aligned}
$$

where $k_{s}^{-}$denotes time $k_{s}$ before the estimate and the FIM are updated. Following Bayes' rule [12], the estimate and the FIM at time $k_{s}$ are updated as

$$
\begin{aligned}
\hat{x}_{k} & =\hat{x}_{k^{-}}+K_{k}\left(y_{k}-H \hat{x}_{k^{-}}\right), \quad k=k_{s}, \\
I_{k} & =I_{k^{-}}+H^{T} R^{-1} H, \quad k=k_{s},
\end{aligned}
$$

where $K_{k}=I_{k}^{-1} H^{T} R^{-1}$ is the gain of the filter.

We write the discrete-time switched dynamics of the estimate and the FIM in terms of the information control:

$$
\begin{aligned}
\hat{x}_{k} & =F \hat{x}_{k-1}+K_{k}\left(y_{k}-H F \hat{x}_{k-1}\right) \delta_{k}, \\
I_{k} & =\left(F I_{k-1}^{-1} F^{T}+Q\right)^{-1}+H^{T} R^{-1} H \delta_{k},
\end{aligned}
$$

which are shortly expressed by $\hat{x}_{k}=\psi_{k}\left(\hat{x}_{k-1}, I_{k-1}, \delta_{k}\right)$ and $I_{k}=\phi_{k}\left(I_{k-1}, \delta_{k}\right)$.

\section{B. Performance Indices}

Entropy is a measure of the uncertainty of a random variable. The differential entropy of the conditional distribution $p\left(x_{k} \mid \mathcal{I}_{k}\left(\delta_{k}\right)\right)$ is given by

$$
H_{k}=\frac{1}{2} \log _{2}\left[(2 \pi e)^{n} \operatorname{det} I_{k}^{-1}\right] .
$$

Definition 3 (Information from decoder's perspective): Information is the change in the entropy of the state of the process given the decoder's information set $\mathcal{I}_{k}\left(\delta_{k}\right)$, i.e.,

$$
\Pi_{k} \triangleq H_{0}-H_{k},
$$

where $H_{0}$ is the entropy at the reference time $k=0$.

In order to optimize the information from decoder's perspective, we define the following estimation cost over the time horizon $N$ :

$$
J_{\pi}^{e}\left(I_{0}\right)=-\frac{1}{2} \sum_{k=0}^{N} \alpha^{k} \log _{2} \operatorname{det} I_{k},
$$

where $\alpha \in(0,1)$ is a discount factor which weighs the relative contribution of the costs in the near and long-term.

The directed communication of the encoder to the decoder is costly. In general, the communication price depends on the state of the channel and on the measurements. Let the communication price per measurement at time $k$ be denoted by $\lambda_{k}$. Then, the communication cost over the time horizon $N$ is given by

$$
J_{\pi}^{c}=\sum_{k=0}^{N} \alpha^{k} \lambda_{k} \delta_{k}
$$

In the following, we assume that $\lambda_{k}=\lambda$ is constant.

We define the aggregate cost function as a convex combination of the estimation and the communication cost functions defined in (14), (15):

$$
J_{\pi}\left(I_{0}\right)=\sum_{k=0}^{N} \alpha^{k} g_{k}\left(I_{k}, \delta_{k}\right),
$$

where the stage cost $g\left(I_{k}, \delta_{k}\right)$ at time $k$ is

$$
g\left(I_{k}, \delta_{k}\right)=-\frac{1}{2} \theta \log _{2} \operatorname{det} I_{k}+(1-\theta) \lambda \delta_{k},
$$


where $\theta \in[0,1]$. The stage cost is a convex function in $I_{k}$ and $\delta_{k}$.

In this study, given the FIM dynamics defined by (11) and the information control defined by (3), we would like to find the optimal stationary sampling policy $\pi^{*}=\left\{\delta^{*}, \delta^{*}, \ldots\right\}$ that minimizes the aggregate cost function $J_{\pi}\left(I_{0}\right)$ when time horizon $N$ goes to infinity.

\section{MAIN RESUlTS}

\section{A. Optimal Stationary Sampling Policy}

The maximal solution $I_{+}$of the FIM dynamics is determined by solving the following equation

$$
\left(F I_{+}^{-1} F^{T}+Q\right)^{-1}+H^{T} R^{-1} H=0 .
$$

The necessary conditions for the existence of a unique maximal solution are studied in [13]. We assume that $I_{+} \succ 0$.

Definition 4: Consider $\gamma>0, \gamma I_{n} \preceq I_{+}$, and $S=\{X \in$ $\left.\mathbb{R}^{n} \times \mathbb{R}^{n} \mid X \succeq \gamma I_{n}\right\}$. We define $U_{\gamma}(I)$ as the set of admissible controls such that if $I \in S$ and $\delta \in U_{\gamma}(I)$, then $\phi(I, \delta) \in S$.

Assumption 1: We assume that the set of admissible controls $U_{\gamma}(I)$ is nonempty for $I \in S$.

Assumption 1 guarantees that the FIM remains a nonsingular matrix given $I_{0} \in S$.

Now, we can formulate the main problem of this paper by the following infinite horizon optimization problem

$$
\begin{array}{ll}
\operatorname{minimize} & J_{\pi}\left(I_{0}\right)=\lim _{N \rightarrow \infty} \sum_{k=0}^{N} \alpha^{k} g\left(I_{k}, \delta_{k}\right) \\
\text { subject to } & I_{k}=\phi\left(I_{k-1}, \delta_{k}\right),
\end{array}
$$

with variables $I_{k} \in S$ and $\delta_{k} \in U_{\gamma}$ for all $k=1,2, \ldots$, and with initial condition $I_{0} \in S$. The optimal value of the cost function in (19) is denoted by $J^{*}\left(I_{0}\right)=\min _{\pi} J_{\pi}\left(I_{0}\right)$.

Definition 5: For any function $J: S \rightarrow \mathbb{R}$, we define the DP operator $T$ as

$$
T J(I)=\min _{\delta \in U_{\gamma}}\{g(I, \delta)+\alpha J(\phi(I, \delta))\},
$$

and the operator $T_{\delta}$ as

$$
T_{\delta} J(I)=g(I, \delta)+\alpha J(\phi(I, \delta)) .
$$

Theorem 1: Given the operators $T$ and $T_{\delta}$ in Definition 5 and Assumption 1, for any bounded function $J: S \rightarrow \mathbb{R}$ we have:

i) The sequence $T^{k} J(I)$ generated by the DP operator is convergent, i.e.,

$$
J^{*}(I)=\lim _{k \rightarrow \infty} T^{k} J(I), \quad \forall I \in S .
$$

ii) The DP operator has a unique fixed point $J^{*}(I)$ that satisfies

$$
J^{*}(I)=T J^{*}(I), \quad \forall I \in S .
$$

iii) The stationary sampling policy $\pi=\{\delta, \delta, \ldots\}$ is optimal if and only if

$$
T_{\delta} J^{*}(I)=T J^{*}(I), \quad \forall I \in S .
$$

Proof: The techniques used for the proof are standard. Part i) For the initial state $I_{0} \in S$ and positive integer $K$, we first split the cost function corresponding to the policy $\pi$ into two parts

$$
J_{\pi}\left(I_{0}\right)=\sum_{k=0}^{K-1} \alpha^{k} g\left(I_{k}, \delta_{k}\right)+\lim _{N \rightarrow \infty} \sum_{k=K}^{N} \alpha^{k} g\left(I_{k}, \delta_{k}\right) .
$$

From Assumption 1, we have $I_{k} \in S$. This leads to $-\frac{1}{2} \theta \log _{2} \operatorname{det} I_{+}+(1-\theta) \lambda \leq g\left(I_{k}, \delta_{k}\right) \leq-\frac{1}{2} \log _{2} \gamma^{n}$. Therefore, there exists $M<\infty$ such that $\left|g\left(I_{k}, \delta_{k}\right)\right| \leq M$. Then, for the second term in (25) we can obtain

$$
\left|\lim _{N \rightarrow \infty} \sum_{k=K}^{N} \alpha^{k} g\left(I_{k}, \delta_{k}\right)\right| \leq \frac{M \alpha^{K}}{1-\alpha} .
$$

Using (26), we can write the following inequalities

$$
\begin{aligned}
& J_{\pi}\left(I_{0}\right)-\frac{M \alpha^{K}}{1-\alpha}-\alpha^{K} \max _{I \in S}|J(I)| \\
& \leq \sum_{k=0}^{K-1} \alpha^{k} g\left(I_{k}, \delta_{k}\right)+\alpha^{K} J\left(I_{K}\right) \\
& \leq J_{\pi}\left(I_{0}\right)+\frac{M \alpha^{K}}{1-\alpha}+\alpha^{K} \max _{I \in S}|J(I)| .
\end{aligned}
$$

Minimizing each term over $\pi$ yields

$$
\begin{aligned}
& J^{*}\left(I_{0}\right)-\frac{M \alpha^{K}}{1-\alpha}-\alpha^{K} \max _{I \in S}|J(I)| \\
& \leq T^{K} J\left(I_{0}\right) \\
& \leq J^{*}\left(I_{0}\right)+\frac{M \alpha^{K}}{1-\alpha}+\alpha^{K} \max _{I \in S}|J(I)| .
\end{aligned}
$$

As $K \rightarrow \infty$, we have $J^{*}\left(I_{0}\right)=\lim _{K \rightarrow \infty} T^{K} J\left(I_{0}\right)$.

Part ii) By applying the DP operator to the equation (28), and from monotonicity of DP, we obtain

$$
\begin{aligned}
& T J^{*}\left(I_{0}\right)-\frac{M \alpha^{K+1}}{1-\alpha}-\alpha^{K+1} \max _{I \in S}|J(I)| \\
& \leq T^{K+1} J\left(I_{0}\right) \\
& \leq T J^{*}\left(I_{0}\right)+\frac{M \alpha^{K+1}}{1-\alpha}+\alpha^{K+1} \max _{I \in S}|J(I)| .
\end{aligned}
$$

As $K \rightarrow \infty$, we know that $J^{*}\left(I_{0}\right)=\lim _{K \rightarrow \infty} T^{K+1} J\left(I_{0}\right)$. Therefore, $J^{*}\left(I_{0}\right)=T J^{*}\left(I_{0}\right)$. Now, assume that $J_{1}$ and $J_{2}$ are two distinct fixed points that satisfy (23). From (22), it follows that $J_{1}^{*}=J_{2}^{*}$.

Part iii) If $T_{\delta} J^{*}(I)=T J^{*}(I)$, from Part ii) we have $J^{*}(I)=T_{\delta} J^{*}(I)$ which yields $J_{\pi}=J^{*}$. The other direction can be proved analogously.

\section{B. Approximate Value and Approximate Policy Iterations}

The state space $S$ of the FIM dynamics is bounded but infinite. In order to use a computational method to solve the DP algorithm, we need to approximate the problem by state discretization. We use a finite grid $S^{\prime}=\left\{I^{1}, \ldots, I^{n\left(S^{\prime}\right)}\right\}$ to discretize the state space where $n\left(S^{\prime}\right)$ denotes the cardinality of $S^{\prime}$. Then, we express the non-grid states by a linear interpolation of grid states, i.e.,

$$
I=\sum_{i=1}^{n\left(S^{\prime}\right)} w_{i}(I) I^{i}
$$


where weights $w_{i}$ are nonnegative. Given that $J\left(I^{i}\right)$ is the value function of a grid state $I^{i}$, the value function of a non-grid state $I$ is approximated by

$$
\tilde{J}(I)=\sum_{i=1}^{n\left(S^{\prime}\right)} w_{i}(I) J\left(I^{i}\right) .
$$

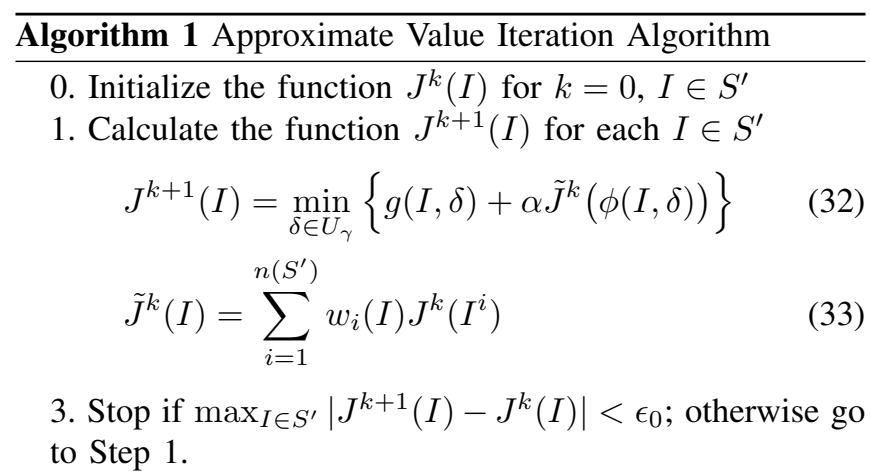

The first method that we use to compute the optimal stationary policy is approximate value iteration (described in Algorithm 1). In approximate value iteration, we generate a sequence of functions $J^{k}(I)$ which are approximations of the functions $T^{k} J(I)$. In each iteration, the improved function $J^{k+1}(I)$ is obtained based on the function $J^{k}(I)$. The algorithm stops when the maximum difference between two successive functions is less than a specific tolerance.

The following proposition guarantees the convergence of the approximate value iteration algorithm.

Proposition 1: Assume that

$$
\max _{I \in S^{\prime}}\left|J^{k+1}(I)-T J^{k}(I)\right| \leq \zeta, \quad k=0,1, \ldots .
$$

Then,

$$
\max _{I \in S^{\prime}}\left|J^{\infty}(I)-J^{*}(I)\right| \leq \frac{\zeta}{1-\alpha},
$$

where $J^{\infty}(I)=\lim _{k \rightarrow \infty} J^{k}(I)$.

Proof: Please refer to [14].

The second method is approximate policy iteration (described in Algorithm 2) that generates a sequence of policies $\pi^{k}=\left\{\delta^{k}, \delta^{k}, \ldots\right\}$ and approximated cost functions $J^{k}(I)$ corresponding to these policies. In each iteration, the cost $J^{k}(I)$ is used by a greedy algorithm to determine the next policy $\pi^{k+1}$. The algorithm stops when the maximum difference between two successive cost functions is less than a tolerance. In comparison with value iteration, policy iteration requires fewer iterations as the policy space is smaller than the state space. However, each iteration in policy iteration is computationally more expensive.

The following proposition guarantees the convergence of the approximate policy iteration algorithm.

Proposition 2: Assume that

$$
\begin{aligned}
& \max _{I \in S^{\prime}}\left|J^{k}(I)-J_{\pi^{k}}(I)\right| \leq \eta, \quad k=0,1, \ldots, \\
& \max _{I \in S^{\prime}}\left|T_{\delta^{k+1}} J^{k}(I)-T J^{k}(I)\right| \leq \zeta, \quad k=0,1, \ldots .
\end{aligned}
$$

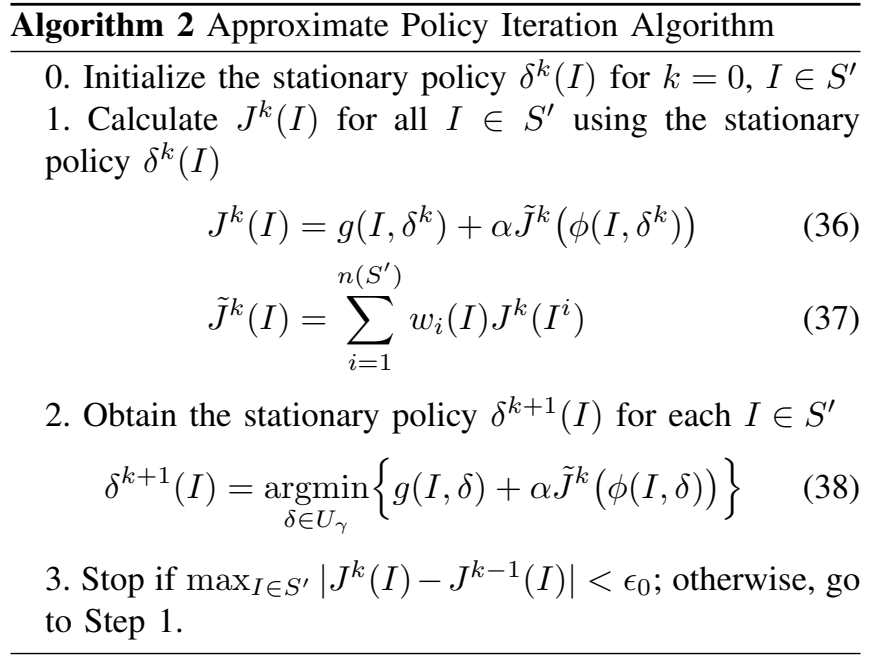

Then,

$$
\max _{I \in S^{\prime}}\left|J_{\pi^{\infty}}(I)-J^{*}(I)\right| \leq \frac{\zeta+2 \alpha \eta}{(1-\alpha)^{2}},
$$

where $J_{\pi^{\infty}}(I)=\lim _{k \rightarrow \infty} J_{\pi^{k}}(I)$.

Proof: Please refer to [14].

Remark 1 (Hybrid Value-Policy Iteration): The cost function $J^{k}(I)$ in Algorithm 2 is obtained iteratively. If the number of iterations for this calculation is moderate, then Algorithm 2 becomes a hybrid of value and policy iterations.

\section{Value of Information and Triggering Mechanisms}

We showed that by using Algorithm 1 or Algorithm 2, we can find the value function and the optimal sampling policy. Here, we will link the optimal policy to the value of information in our system, and we will propose two triggering mechanisms that can generate the optimal policy.

Definition 6 (Value of information): The value of information (VoI) is the maximum value that the observer would be willing to pay for the sampling and the transmission of a measurement $y_{k}$ at time $k$, i.e.,

$$
\operatorname{VoI}\left(I_{k}\right)=J^{*}\left(\phi\left(I_{k}, 0\right)\right)-J^{*}\left(\phi\left(I_{k}, 1\right)\right),
$$

where $J^{*}$ is the value function.

In the following, we introduce two triggering mechanisms based on the value of information and on the FIM (or covariance) threshold that can generate the optimal policy.

Proposition 3: Let VoI $: S \rightarrow \mathbb{R}$ be the value of information function. Then, the sampling policy specified by the triggering mechanism

$$
\delta(I)= \begin{cases}1, & \text { if } \alpha \operatorname{VoI}(I) \geq(1-\theta) \lambda, \\ 0, & \text { otherwise, }\end{cases}
$$

is optimal for all $I$ that $\phi(I, \delta) \in S$.

Proof: From the Bellman equation, the optimal stationary sampling policy is given by

$$
\delta^{*}(I)=\underset{\delta \in U_{\gamma}}{\operatorname{argmin}}\left\{g(I, \delta)+\alpha J^{*}(\phi(I, \delta))\right\} .
$$

This implies that $\delta^{*}(I)=1$ if and only if $g(I, 0)+$ $\alpha J^{*}(\phi(I, 0)) \geq g(I, 1)+\alpha J^{*}(\phi(I, 1))$. Substituting $g(I, 0)$ 
and $g(I, 1)$ from the equation (17) in the inequality, we obtain $\alpha J^{*}(\phi(I, 0))-\alpha J^{*}(\phi(I, 1)) \geq(1-\theta) \lambda$. From Definition 6, it follows that $\alpha \operatorname{VoI}(I) \geq(1-\theta) \lambda$.

Definition 7: A function $F(x, y): X \times Y \rightarrow \mathbb{R}$ has non-decreasing differences in $(x, y)$ if for any $x_{1}, x_{2} \in X$, $y_{1}, y_{2} \in Y, x_{2} \geq x_{1}$, and $y_{2} \geq y_{1}$ we have

$$
F\left(x_{2}, y_{2}\right)+F\left(x_{1}, y_{1}\right) \geq F\left(x_{2}, y_{1}\right)+F\left(x_{1}, y_{2}\right)
$$

Theorem 2: Assume that the system (1), (2) is scalar. There exists a threshold $\tau>0$ such that the sampling policy specified by the triggering mechanism

$$
\delta(I)= \begin{cases}1, & \text { if } I \leq \tau, \\ 0, & \text { otherwise }\end{cases}
$$

is optimal for all $I$ that $\phi(I, \delta) \in S$.

Proof: Define $\Gamma(I, \delta)=g(I, \delta)+\alpha J^{*}(\phi(I, \delta))$. We will show that $\Gamma(I, \delta)$ has non-decreasing differences in $(I, \delta)$ for all $I \in S$ and $\delta \in U_{\gamma}$. Since $g(I, \delta)$ is a separable function, it is enough to show that $J^{*}(\phi(I, \delta))$ has non-decreasing differences in $(I, \delta)$. To this purpose, we will first prove by induction that $J^{*}(I)$ with the domain $S$ is convex.

We can choose $T^{0} J(I)=0$. Assume that $T^{k} J(I)$ is convex in the domain $S$. We will show that $T^{k+1} J(I)$ is convex. From Definition 5, we have

$$
T^{k+1} J(I)=\min _{\delta \in U_{\gamma}}\left\{g(I, \delta)+\alpha T^{k} J(\phi(I, \delta))\right\},
$$

which for the minimizer $\delta^{+}$yields

$$
T^{k+1} J(I)=g\left(I, \delta^{+}\right)+\alpha T^{k} J\left(\phi\left(I, \delta^{+}\right)\right) .
$$

However, $g\left(I, \delta^{+}\right)$is convex by definition (17), and by the inductive hypothesis $T^{k} J(I)$ is convex. Hence, $T^{k+1} J(I)$ is convex. According to Theorem 1-i for any initial function $T^{0} J(I)$, we have

$$
J^{*}(I)=\lim _{k \rightarrow \infty} T^{k+1} J(I), \quad \forall I \in S .
$$

This implies that $J^{*}(I)$ is convex.

Now, assume that $I^{\prime \prime} \geq I^{\prime}$ and $t \geq 0$. We can find $\theta^{\prime}, \theta^{\prime \prime} \in$ $[0,1]$ such that $\theta^{\prime}+\theta^{\prime \prime}=1, I^{\prime}+t=\left(1-\theta^{\prime}\right) I^{\prime}+\theta^{\prime}\left(I^{\prime \prime}+t\right)$, and $I^{\prime \prime}=\left(1-\theta^{\prime \prime}\right) I^{\prime}+\theta^{\prime \prime}\left(I^{\prime \prime}+t\right)$. By the convexity of $J^{*}(I)$, we can write

$$
\begin{aligned}
& J^{*}\left(I^{\prime}+t\right) \leq\left(1-\theta^{\prime}\right) J^{*}\left(I^{\prime}\right)+\theta^{\prime} J^{*}\left(I^{\prime \prime}+t\right), \\
& J^{*}\left(I^{\prime \prime}\right) \leq\left(1-\theta^{\prime \prime}\right) J^{*}\left(I^{\prime}\right)+\theta^{\prime \prime} J^{*}\left(I^{\prime \prime}+t\right) .
\end{aligned}
$$

Combining the equations (50) and (51), we conclude

$$
J^{*}\left(I^{\prime \prime}+t\right)-J^{*}\left(I^{\prime \prime}\right) \geq J^{*}\left(I^{\prime}+t\right)-J^{*}\left(I^{\prime}\right) .
$$

From Definition 7, the function $J^{*}(\phi(I, \delta))$ has nondecreasing differences in $(I, \delta)$ if for all $I_{2} \geq I_{1}$, we have

$J^{*}\left(\phi\left(I_{2}, 1\right)\right)-J^{*}\left(\phi\left(I_{2}, 0\right)\right) \geq J^{*}\left(\phi\left(I_{1}, 1\right)\right)-J^{*}\left(\phi\left(I_{1}, 0\right)\right)$.

Using the definition of $\phi(I, \delta)$ in (11), we can define

$$
\begin{aligned}
& I^{\prime}=\phi\left(I_{1}, 0\right)=\left(F I_{1}^{-1} F^{T}+Q\right)^{-1}, \\
& I^{\prime \prime}=\phi\left(I_{2}, 0\right)=\left(F I_{2}^{-1} F^{T}+Q\right)^{-1}, \\
& t=H^{T} R^{-1} H>0 .
\end{aligned}
$$

Hence, $I^{\prime \prime} \geq I^{\prime}$ provided that $I_{2} \geq I_{1}$. By rewriting the equation (53) in terms of $I^{\prime}, I^{\prime \prime}$, and $t$, we have

$$
J^{*}\left(I^{\prime \prime}+t\right)-J^{*}\left(I^{\prime \prime}\right) \geq J^{*}\left(I^{\prime}+t\right)-J^{*}\left(I^{\prime}\right)
$$

which we already showed that holds for $I^{\prime \prime} \geq I^{\prime}$ and $t \geq 0$. Therefore, $J^{*}(\phi(I, \delta))$ has non-decreasing differences in $(I, \delta)$. This means that $\Gamma(I, \delta)$ has also non-decreasing differences in $(I, \delta)$.

From the Bellman equation, the optimal stationary sampling policy is given by

$$
\delta^{*}(I)=\underset{\delta \in U_{\gamma}}{\operatorname{argmin}}\left\{g(I, \delta)+\alpha J^{*}(\phi(I, \delta))\right\} .
$$

From Assumption 1, the minimizer $\delta^{*}(I)$ always exists for all $I \in S$. Furthermore, following Topkis's monotonicity theorem [15] since $\Gamma(I, \delta)=g(I, \delta)+\alpha J^{*}(\phi(I, \delta))$ has non-decreasing differences in $(I, \delta)$, the minimizer $\delta^{*}(I)$ is monotonically non-increasing in $I$. In addition, since $\delta$ can be 0 or 1 there exists a threshold $\tau>0$ such that $\delta^{*}(I)=1$ for all $I \leq \tau$, and $\delta^{*}(I)=0$ otherwise. Therefore, the threshold policy is the optimal sampling policy.

Corollary 1: The value of information function VoI : $S \rightarrow \mathbb{R}$ is a monotonically non-increasing function.

Proof: Following Theorem 2, from the equation (53) and Definition 6 for any $I_{1}, I_{2} \in S, I_{2} \geq I_{1}$ we have

$$
\operatorname{VoI}\left(I_{1}\right) \geq \operatorname{VoI}\left(I_{2}\right) \text {. }
$$

This implies that $\operatorname{VoI}(I)$ is a monotonically non-increasing function.

Remark 2: The relation between the the value of information function $\operatorname{VoI}(I)$ and the FIM threshold $\tau$ is given by $\alpha \operatorname{VoI}(\tau)-(1-\theta) \lambda=0$.

\section{ILLUSTRATIVE EXAMPLE}

In this section, we present numerical and simulation results for a simple unstable system.

Example 1: Consider a system with the following linear scalar dynamics

$$
\begin{aligned}
& x_{k}=1.01 x_{k-1}+w_{k-1}, \\
& y_{k}=0.3 x_{k}+v_{k},
\end{aligned}
$$

where the covariances are $Q=0.05$ and $R=1.1$. It is assumed that $\alpha=0.95, \theta=0.5$, and the FIM space is discretized by a grid with the cardinality $n\left(S^{\prime}\right)=1000$. The initial condition for the FIM is $I_{0}=1$. The results are provided for two different prices $\lambda=\{0.5,0.9\}$.

Figure 1 shows the value functions $J^{*}(I)$ for the two prices. These functions in accordance with what we derived in Theorem 2 have non-decreasing differences. Figure 2 shows the values of information for the two prices. As it is seen, they are monotonically non-increasing. The critical values are $\operatorname{VoI}(\tau)=0.2632$ and $\operatorname{VoI}(\tau)=0.4738$, and the corresponding FIM thresholds are $\tau=0.8163$ and $\tau=0.5194$, respectively for $\lambda=0.5$ and $\lambda=0.9$. Figure 3 illustrates the information diagrams for the two prices when the optimal stationary sampling policies are applied. The total number of samplings over the horizon $N=100$ are 


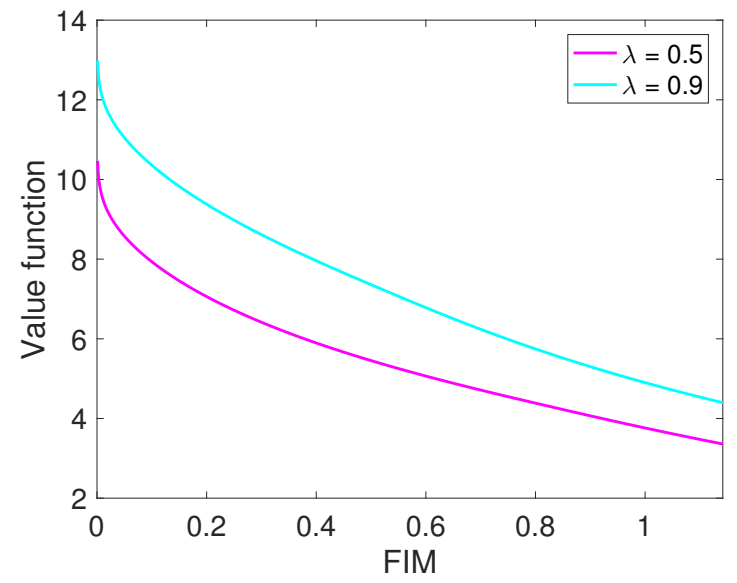

Fig. 1. Value functions, $J^{*}(I)$.

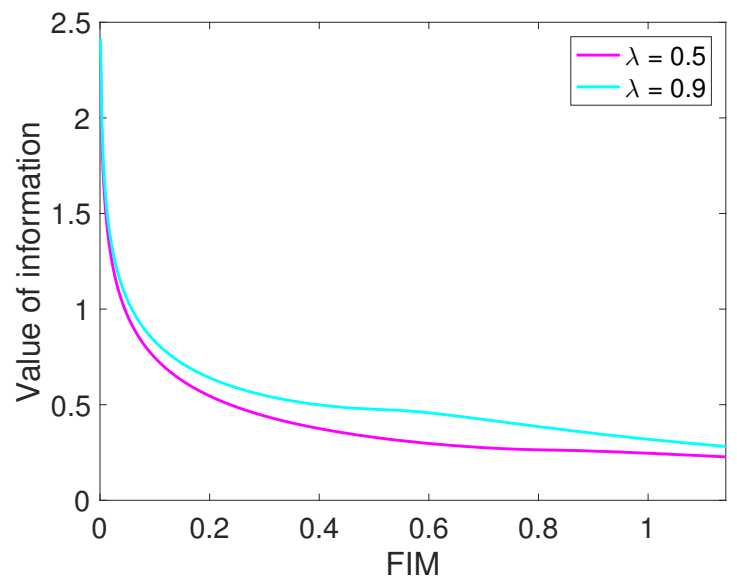

Fig. 2. Value of information functions, $\operatorname{VoI}(I)$.

$M=55$ and $M=26$, respectively for $\lambda=0.5$ and $\lambda=0.9$. As it is expected, the total number of samplings decreases when the communication price increases.

\section{CONCLUSION}

In this paper, we developed a framework for obtaining the optimal stationary sampling policy based on the FIM dynamics for transmission of measurements of a stochastic process from an encoder to a decoder through a costly communication channel. We proposed two computational methods, i.e., value iteration and policy iteration, to compute the optimal policy. We showed that there exists a triggering mechanism based on the value of information that generates the optimal policy. In addition, we showed that due to the monotonic property of the value of information, there exists a triggering mechanism based on the FIM threshold that specifies the optimal policy for scalar systems.

\section{ACKNOWLEDGMENT}

This work has been carried out with the support of the Technische Universität München - Institute for Advanced Study, funded by the German Excellence Initiative and the DFG Priority Program SPP1914 "Cyber-Physical Networking". The research of J. S. Baras was supported by AFOSR

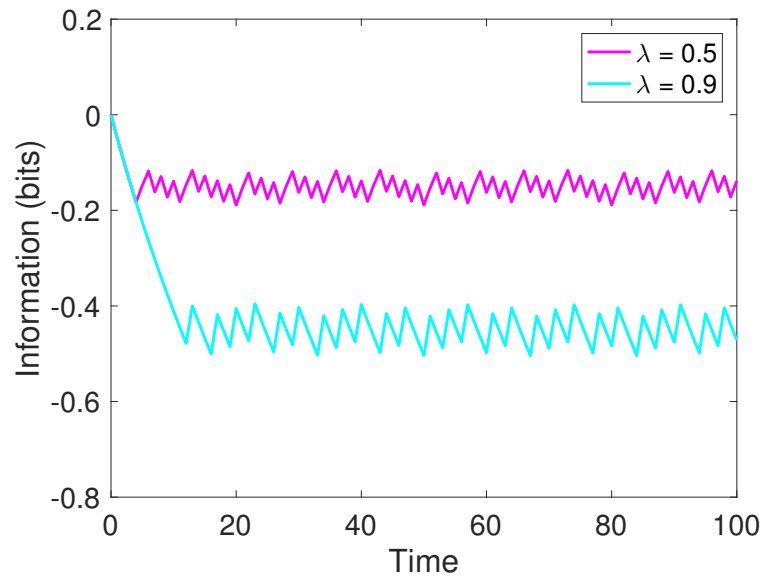

Fig. 3. Information, П, diagrams corresponding to the optimal policies.

MURI grant FA9550-09-1-0538, by DARPA through ARO grant W911NF1410384, by ARO grant W911NF-15-1-0646, by NSF grant CNS-1544787 and by a Senior Hans Fisher Fellowship from IAS-TUM.

\section{REFERENCES}

[1] J. Mark and T. Todd, "A nonuniform sampling approach to data compression," IEEE Trans. Communications, vol. 29, no. 1, pp. 24-32, 1981.

[2] K. J. Åström and B. Bernhardsson, "Comparison of Riemann and Lebesgue sampling for first order stochastic systems," in Proc. of the IEEE Conf. on Decision and Control, pp. 2011-2016, 2002.

[3] H. J. Kushner, "On the optimum timing of observations for linear control systems with unknown initial state," IEEE Trans. Automatic Control, vol. 9, no. 2, pp. 144-150, 1964.

[4] L. Meier, J. Peschon, and R. M. Dressler, "Optimal control of measurement subsystems," IEEE Trans. Automatic Control, vol. 12, no. 5, pp. 528-536, 1967.

[5] M. Rabi, G. V. Moustakides, and J. S. Baras, "Adaptive sampling for linear state estimation," SIAM Journal on Control and Optimization, vol. 50, no. 2, pp. 672-702, 2012.

[6] A. Molin and S. Hirche, "An iterative algorithm for optimal eventtriggered estimation," in Proc. of the IFAC Conf. on Ana. and Design of Hybrid Sys., pp. 64-69, 2012.

[7] T. Soleymani, S. Hirche, and J. S. Baras, "Optimal information control in cyber-physical systems," in Proc. of the IFAC Workshop on Distrib. Estimation and Control in Netw. Syst., 2016.

[8] W. Heemels, K. H. Johansson, and P. Tabuada, "An introduction to event-triggered and self-triggered control," in Proc. of the IEEE Conf. on Decision and Control, pp. 3270-3285, 2012.

[9] T. Soleymani, S. Hirche, and J. S. Baras, "Maximization of information in energy-limited directed communication," in Proc. of the European Control Conf., 2016.

[10] T. Soleymani, S. Hirche, and J. S. Baras, "Optimal self-driven sampling for estimation based on value of information," in Proc. of the Int. Workshop on Discrete Event Systems, pp. 183-188, 2016.

[11] D. Guo, S. Shamai, and S. Verdú, "The interplay between information and estimation measures," Foundations and Trends in Signal Processing, vol. 6, no. 4, pp. 243-429, 2012.

[12] K. J. Åström, Introduction to stochastic control theory. Dover Publications, 2006.

[13] K. Zhou, J. C. Doyle, K. Glover, et al., Robust and optimal control, vol. 40. Prentice hall New Jersey, 1996.

[14] D. P. Bertsekas, Dynamic programming and optimal control. Athena Scientific Belmont, MA, 1995.

[15] D. M. Topkis, "Minimizing a submodular function on a lattice," Operations research, vol. 26, no. 2, pp. 305-321, 1978. 